\title{
Special Modes in Spin Wave Spectra of Two-Dimensional Nanodots
}

\begin{abstract}
S. MAMICA*
Faculty of Physics, A. Mickiewicz University in Poznań, Umultowska 85, 61-614 Poznań, Poland

We use a microscopic theory taking into account the nearest-neighbour exchange and dipolar interactions to study two-dimensional magnetic nanodots and nanorings. Magnetic configuration is assumed to form an inplane vortex (circular magnetization). We examine the dependence of the frequencies and profiles of spin waves on the dipolar-to-exchange interaction ratio $d$ and the size of the dot (ring). Special attention is paid to some particular modes, including the lowest mode in the spectrum and the fundamental mode, the frequency of which proves almost independent of $d$. In the case of the lowest mode different profiles are observed: azimuthal, fundamental (quasiuniform) or highly localized, depending on $d$ and the size. We also study the fundamental mode evolution including its hybridization and explain the selection rules.
\end{abstract}

DOI: 10.12693 /APhysPolA.127.365

PACS: 75.30.Ds, 75.70.Kw, 75.75.Jn

\section{Introduction}

Concurrence of the short and long range interactions is responsible for a number of effects in a variety of systems [1-5]. In small magnetic dots the competition between the exchange and dipolar interactions leads to a rich spectrum of stable and metastable magnetic configurations, including vortex states $[6,7]$. Potential applications of magnetic vortices include single magnetic nanoparticle sensing and trapping [8], microwavefrequency oscillators [9], data storage and information processing [10], or frequency multiplication [11]. An important role in all of these applications is played by spin waves. In the present study we focus on the spin-wave spectrum of two-dimensional (2D) circular dots and rings with particular attention paid on two special modes: the lowest mode in the spectrum and the fundamental mode.

The lowest mode plays a special role in metastable vortices, in which it becomes a soft mode responsible for the transition to a different magnetic configuration [12]. In experimental and micromagnetic studies the lowest spin-wave mode is reported to be an azimuthal mode of different order [13], a localized mode [14], or even the fundamental mode [15]. Here we show that for a critical value of the dipolar-to-exchange interaction ratio $d$, for which the vortex state loses its (meta)stability, the lowest mode can have three forms: localized, delocalized but confined to a small area in the ring, or almost uniform. Beyond this critical situation, the lowest mode is an azimuthal mode of the order depending on $d$.

We also analyze the fundamental mode evolution as a function of $d$. We show that its symmetry increases with growing $d$ which results in occurrence of several anticrossings with different azimuthal modes. The latest studies show a major role of mode hybridization in plasmonic devices [16]. The issue also affects the profiles of

*e-mail: mamica@amu.edu.pl the hybridizing modes, which is important in the context of the influence of the internal excitations of nanoparticles on their magnetization reversal, even for nanoparticles smaller than the exchange length [17].

\section{Theoretical approach}

The object is a circular dot cut out of a $2 \mathrm{D}$ square lattice with elementary magnetic moments (spins) in its sites. Since the dot is based on a discrete lattice its boundary is not perfectly circular, thus by "circular" we mean cut with a circle. With increasing the diameter the boundary of the dot becomes smoother, which has an effect on the spin-wave profiles, but general features remain unchanged even in rather small dots.

We consider the dynamics of a magnetic moment $\boldsymbol{M}_{\boldsymbol{R}}$, $\boldsymbol{R}$ being the position vector, in the linear approximation, assuming $\left|\boldsymbol{m}_{\boldsymbol{R}}\right| \ll\left|\boldsymbol{M}_{\boldsymbol{R}}\right|,\left|\boldsymbol{M}_{0, \boldsymbol{R}}\right| \approx\left|\boldsymbol{M}_{\boldsymbol{R}}\right|$ and $\boldsymbol{m}_{\boldsymbol{R}} \perp \boldsymbol{M}_{\boldsymbol{R}}$ where $\boldsymbol{M}_{0, \boldsymbol{R}}$ and $\boldsymbol{m}_{\boldsymbol{R}}$ are the static and dynamic component of the magnetic moment, respectively. To describe the time evolution of $\boldsymbol{m}_{\boldsymbol{R}}$, oscillating harmonically with a frequency $\omega$, we use the damping-free Landau-Lifshitz (LL) equation taking into account the dipolar and exchange interactions. After linearization of the LL equations we obtain a system of equations for the in-plane and out-of-plane coordinates of the dynamic component of the magnetic moments. Numerical diagonalization of the corresponding eigenvalue problem yields the frequency spectrum of the spin-wave excitations, and the spin-wave profiles, i.e., the distribution of the inplane $\left(m_{r}\right)$ and out-of-plane $\left(m_{k}\right)$ amplitudes of precession of the elementary magnetic moments. (For more details please see our paper [18].)

There is only one material parameter used in our model, namely the dipolar-to-exchange interaction ratio

$$
d=\frac{\left(g \mu_{\mathrm{B}}\right)^{2} \mu_{0}}{8 \pi a_{\mathrm{NN}}^{3} J},
$$

where $g$ is the g-factor, $\mu_{\mathrm{B}}-$ the Bohr magneton, $\mu_{0}$ the vacuum permeability, $a_{\mathrm{NN}}$ - the nearest-neighbour distance, and $J$ - the nearest-neighbour exchange integral. In the case of square and hexagonal lattices $a_{\mathrm{NN}}$ is the lattice constant. 
There are no simulations performed in this approach. The magnetic configuration is assumed to be the in-plane vortex: the static component of each magnetic moment in the system lies in the plane of the dot perpendicularly to its radius. We receive the information about its stability from the spin wave spectrum. The occurrence of zero-frequency excitations is indicative of the presence of nucleation modes and instability of the assumed magnetic state. The lack of zero-frequency mode implies the (meta)stability of the assumed magnetic configuration.

\section{Results}

In Fig. 1 we show a typical dependence of the spinwave spectrum on the dipolar-to-exchange interaction ratio $d$ for a circular dot. The dependence clearly indicates the existence of three ranges of $d$ : below $d_{1}$ and above $d_{2}$ the assumed in-plane vortex is not stable, while between these critical values absence of the zerofrequency modes indicates its (meta)stability. (The problem of the in-plane vortex stability has been studied in our papers [18-20].)

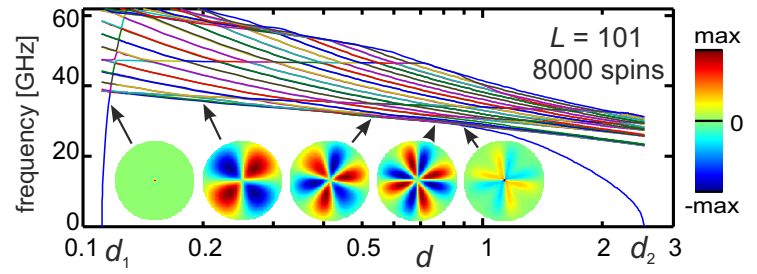

Fig. 1. Spin-wave frequency of 36 lowest modes vs. $d$ (in logarithmic scale) for an in-plane vortex state in 2D circular dot of diameter $L=101$ lattice constants. The lack of zero-frequency modes indicates the stability of the assumed magnetic state for $d_{1}<d<d_{2}$. Also the profiles of the lowest mode for five values of $d$ marked with arrows are shown.

The lowest mode evolution as a function of $d$ for circular dots was studied in our paper [12] thus here let us recall only most important results. Just above $d_{1}$ the lowest mode is strongly localized at the dot centre; at the same time its frequency increases steeply with increase of $d$. Such behaviour is typical for a soft mode: as $d$ continues to grow the excitation of this mode becomes increasingly difficult. For $d \approx 0.1171$ the localized mode crosses the second order azimuthal mode $(0,2)$. The frequency of azimuthal modes decreases with growing $d$ with the rate which depends on their azimuthal number, for higher order modes it decreases faster. Thus for $d>0.37$ mode $(0,3)$ is the lowest in the spectrum and for $d \approx 0.68$ mode $(0,4)$ starts to be the lowest one. Above $d \approx 0.82$ we observe softening of the lowest mode and again localized mode has the lowest frequency but now it has azimuthal number 2 .

As we show in [12] in dots smaller than 100 lattice constants in diameter for small $d$ the lowest-frequency mode is first order azimuthal mode while for higher $d$ mode $(0,2)$. It stay the lowest in the spectrum until $d=d_{2}$ continuously localizing at the centre. We show the dependence of the frequency $f$ of the lowest mode vs. the diameter $L$ of the dot to be approximately $f \sim L^{1 / 2}$, which is in good agreement with analytical study [21, 22]. For a fixed material (given $d$ ) increase of the size of the dot results in increase of the order of the lowest azimuthal mode; such effect was observed experimentally [13] and analytically [22].

The size of a dot has no influence on both $d_{1}$ and the $f(d)$ of the lowest mode in vicinity of $d_{1}$ until it crosses an azimuthal mode. We found different situation in circular rings [20] where removing of the central part of a dot results in lack of lowest mode localization.

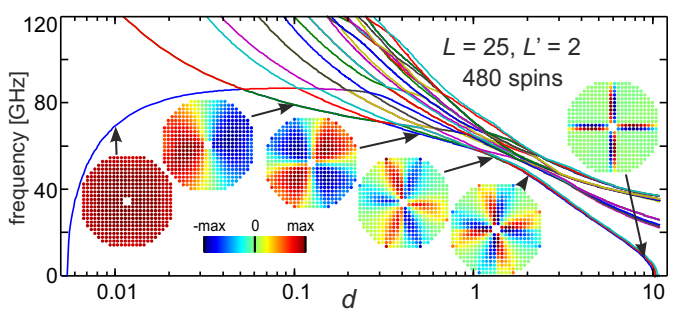

Fig. 2. Spin-wave frequency of 25 lowest modes vs. $d$ (in logarithmic scale) for an in-plane vortex state in $2 \mathrm{D}$ ring of external diameter $L=25$ and internal one $L^{\prime}=2$ lattice constants. The profiles of the lowest mode for six values of $d$ marked with arrows are shown.

In Fig. 2 we show the spin-wave spectrum vs. $d$ for the ring of outer diameter $L=25$ lattice constants and inner one $L^{\prime}=2$, which means that only four spins are removed from the dot centre. The plot is accompanied by the profiles of the lowest spin-wave mode, corresponding to the points marked with arrows. The external size is much lower than the mentioned above threshold 100 lattice constants but the lowest mode behaviour changed dramatically. There is no more strongly localized mode in the spectrum: near $d_{1}$ the fundamental mode is the lowest. While $d$ increases, successive azimuthal modes become the lowest up to $(0,4)$. It is similar to dots with $L>100$ and results from weakening exchange interaction due to removal of the vortex centre. Mode $(0,4)$ remains the lowest mode until $d_{2}$ with increasing localization at the high spin density lines.

Another special mode is the fundamental mode $(0,0)$ with the frequency very little dependent on $d$. The evolution of this mode is shown in Fig. 3. Its profile is not uniform (Fig. 3b, $d=0.112$ ) because the dot is based on the square lattice. (Similar effect occurs for micromagnetic simulations due to the artificial discretization of the sample [15].) As we show in [12], it has two consequences for the fundamental mode behaviour: its frequency changes with $d$ (it should not for a uniform excitation) and it hybridizes with the azimuthal mode of the same symmetry. Additionally, for $d \approx d_{1}$ we observe hybridization of three modes: localized, azimuthal $(0,4)$ and fundamental (see mixed profiles for $d=0.133$ ).

After hybridization the profile of the fundamental mode is restored $(d=0.235)$ but rotated by $\pi / 4$ with maxima along the high spin density lines. While $d$ continues to increase these maxima split and the symmetry 


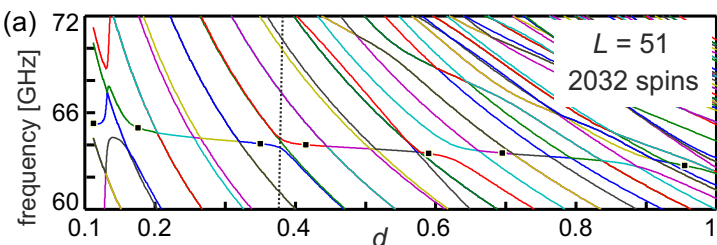

(b)
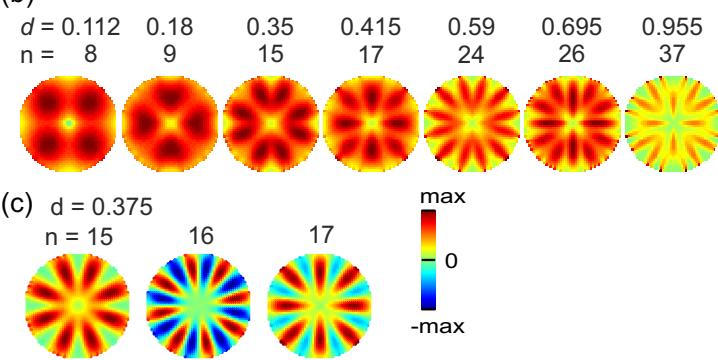

Fig. 3. (a) Evolution of the fundamental mode vs. $d$ for the dot of the diameter $L=51$. (b) Profiles of the fundamental mode for points marked with black squares in (a). (c) Profiles of hybridizing modes for $d=0.375$. Also, the profile of the second $(0,8)$ mode which is not involved in the hybridization is shown.

is doubled $(d=0.35)$, thus another hybridization occurs with $(0,8)$ mode $(d=0.35-0.415)$. This effect continues with growing $d$ and next two hybridizations occur. For $d>1$ the mode under the question loses its fundamental character; its profile has pronounced maxima and minima, and its frequency noticeably depends on $d$.

In every case of hybridization only one azimuthal mode of a given symmetry is involved while the other one is "ignored" (see the profiles in Fig. 3c). This is because the amplitude maxima of the fundamental mode coincide with the nodal lines in its profile. Thus, both identical symmetry and the coincidence of the anti-nodes of the same phase of the azimuthal mode with the amplitude maxima of the fundamental mode are necessary for mode hybridization to occur. (The same rules hold for multi-mode hybridization [12].)

\section{Conclusions}

The lowest-frequency mode has different character depending on the stability of the vortex state. Far from the critical value of the dipolar-to-exchange interaction ratio $d$ azimuthal modes are the lowest with azimuthal number $m$ being the compromise between exchange and dipolar interactions: exchange interactions prefer lower $m$ while dipolar interactions favour higher $m$, regardless of whether their predomination is due to the material or size of the dot (ring). Close to the critical value of $d$ the localized mode is the lowest in dots and the quasi-uniform one in rings (compare our papers $[20,23])$. Thus the profile of the lowest mode carry information on the stability of the vortex.

The profile of the fundamental mode, an analogue of the uniform mode, has the symmetry inherited from the lattice the dot is based on (compare results for hexagonal lattice [23]). Additionally, its symmetry grows with $d$. As a consequence, with growing $d$, it hy- bridizes with descending azimuthal modes of the corresponding symmetry. Additional condition is coincidence of the maxima of the fundamental mode with the antinodal lines of the same phase of the azimuthal mode.

\section{Acknowledgments}

This study has received financial support from the European Community's 7th Framework Programme, Grant Agreement No. 247556 (NoWaPhen) and from the National Science Centre (NCN) of Poland (Project No. DEC-2012/07/E/ST3/00538).

\section{References}

[1] H. Puszkarski, J.-C. Lévy, S. Mamica, Phys. Lett. A 246, 347 (1998).

[2] S. Mamica, H. Puszkarski, J.-C. Lévy, Phys. Status Solidi B 218, 561 (2000).

[3] M. Krawczyk, H. Puszkarski, J.-C. Lévy, S. Mamica, D. Mercier, J. Magn. Magn. Mater. 246, 93 (2002).

[4] S. Mamica, M. Krawczyk, J. Kłos, Adv. Condens. Matter Phys. 2012, 161387 (2012).

[5] S. Mamica, M. Krawczyk, M.L. Sokolovskyy, J. Romero-Vivas, Phys. Rev. B 86, 144402 (2012).

[6] K.L. Metlov, Y.P. Lee, Appl. Phys. Lett. 92, 112506 (2008).

[7] Ph. Depondt, J.-C.S. Lévy, S. Mamica, J. Phys. Condens. Matter 25, 466001 (2013).

[8] M. Donolato, M. Gobbi, P. Vavassori, M. Leone, M. Cantoni, V. Metlushko, B. Ilic, M. Zhang, S.X. Wang, R. Bertacco, Nanotechnology 20, 385501 (2009).

[9] K.Y. Guslienko, J. Spintron. Magn. Nanomater. 1, 70 (2012).

[10] R.P. Cowburn, M.E. Welland, Science 287, 1466 (2000).

[11] V.E. Demidov, H. Ulrichs, S. Urazhdin, S.O. Demokritov, V. Bessonov, R. Gieniusz, A. Maziewski, Appl. Phys. Lett. 99, 012505 (2011).

[12] S. Mamica, J.-C.S. Lévy, M. Krawczyk, J. Phys. D 47, 015003 (2014).

[13] M. Buess, T.P.J. Knowles, R. Hollinger, T. Haug, U. Krey, D. Weiss, D. Pescia, M.R. Scheinfein, C.H. Back, Phys. Rev. B 71, 104415 (2005).

[14] X. Zhu, Z. Liu, V. Metlushko, P. Grutter, M.R. Freeman, Phys. Rev. B 71, 180408(R) (2005).

[15] R. Wang, X. Dong, Appl. Phys. Lett. 100, 082402 (2012).

[16] D. Ju, Y. Jiang, H. Pei, Plasmonics 8, 1387 (2013).

[17] S. Rohart, P. Campiglio, V. Repain, Y. Nahas, C. Chacon, Y. Girard, J. Lagoute, A. Thiaville, S. Rousset, Phys. Rev. Lett. 104, 137202 (2010).

[18] S. Mamica, J.-C.S. Lévy, M. Krawczyk, Ph. Depondt, J. Appl. Phys. 112, 043901 (2012).

[19] S. Mamica, J.-C.S. Lévy, Ph. Depondt, M. Krawczyk, J. Nanopart. Res. 13, 6075 (2011).

[20] S. Mamica, J. Appl. Phys. 113, 093901 (2013).

[21] B.A. Ivanov, C.E. Zaspel, Phys. Rev. Lett. 94, 027205 (2005).

[22] R. Zivieri, F. Nizzoli, Phys. Rev. B 71, 014411 (2005).

[23] S. Mamica, J. Appl. Phys. 114, 233906 (2013). 